\title{
Effect of Planting Patterns and Nk Fertilizers Levels under Two Planting Date on Yield and Yield Components of Egyptian cotton In Middle Egypt
}

Ghoprial M. A. S. *, S. A. Sedhom**, M. A. A. Emara*, E. M. M. El-Gedwy** and S. A. S. Mehasen ${ }^{1 * *}$

* Cotton Research Institute, Agricultural Research Center, Giza, Egypt

**Agronomy Department, Faculty of Agriculture, Benha University, Egypt

${ }^{1}$ Corresponding author: Sadiek Abdelaziz Sadiek Mehasen, Head Department of Agronomy, Faculty of Agriculture at Moshtohor, Benha University, Egypt. PO Box 13736.

\begin{abstract}
Two separate field experiments were conducted during 2019 and 2020 seasons, at Mallawi region, Minia Governorate, Egypt, to study the effect of two planting date, four planting patterns and three NK fertilizer levels on yield and yield components of Egyptian cotton (Gossypium barbadense, L.) promising hybrid cotton (CB58 X Giza 90). The results could be summarized as follows:

Planting date at $20^{\text {th }}$ March was significantly increase of No. of fruiting branches plant ${ }^{-1}$, No. of open bolls plant $^{-1}$, Boll weight, Seed index, Lint (\%), Seed cotton yield plant ${ }^{-1}$, Seed cotton yield fed ${ }^{-1}$ in both seasons. Whereas, planting date at $20^{\text {th }}$ April were significantly increase of Plant height, in two season. Regarding planting patterns was significantly effect on plant height, No. of fruiting branches plant ${ }^{-1}$, No. of open bolls plant ${ }^{-1}$, Boll weight, Seed index, Lint (\%), Seed cotton yield plant ${ }^{-1}$, Seed cotton yield fed ${ }^{-1}$ in the both seasons. Concerning NK fertilizer levels had a significant effect on plant height, No. of fruiting branches plant ${ }^{-1}$, No. of open bolls plant $^{-1}$, Boll weight, Seed index, Lint (\%), Seed cotton yield plant ${ }^{-1}$, Seed cotton yield fed ${ }^{-1}$ in the both seasons. Boll weight, seed index and seed cotton yield fed ${ }^{-1}$ in the first season and seed cotton yield plant ${ }^{-1}$ in the second season were affected by the interaction between planting date and planting patterns. Moreover, plant height and No. open bolls plant ${ }^{-1}$ were affected by the interaction between planting date and NK fertilizer levels in the first and second seasons, respectively. While, plant height and No. open bolls plant ${ }^{-1}$ were affected by the interaction between planting patterns and NK fertilizer levels in the first and second seasons, respectively. Regarding the interaction effect, among planting date, planting patterns and NK fertilizer levels were significant differences of No. fruiting branches plant ${ }^{-1}$ in the first season only. It can be concluded that early planting, $130 \mathrm{~cm}$ bed width + $30 \mathrm{~cm}$ hill space planted in two sides and fertilized by $75 \mathrm{~kg} \mathrm{~N}+48 \mathrm{~kg} \mathrm{~K}_{2} \mathrm{O}$ fed $^{-1}$ under the conditions of the experiment.
\end{abstract}

Key Words: Planting dates, planting patterns, NK fertilizer levels, yield and yield components, Egyptian cotton

\section{Introduction}

Egyptian cotton (Gossypium barbadense, L.) is the most important commercial fiber crop in Egypt. Cotton plays a key role in economic activity. It is the oldest among the commercial crops and is regarded as white gold. Egyptian cotton is preferred around the world because it is long fiber cotton that makes it softer and stronger at the same time. For many years, it was so valuable that most of the crop was exported to European countries. Cotton seed meal is used in food products for animal feed due to its high protein and energetic values. So, it is necessary to increase cotton cultivation area and productivity. Cotton is not only the most important fiber crop of the world but also the second best source for plant proteins after soybean and the oil ranking fifth in the world use among edible oils (Sawan et al, 2006). Any other economic characters are influenced by the various agronomic treatments especially planting date, planting patterns the cotton plants the amount of fertilizers.

In this connection, planting date differ in cotton yield and yield attributes as reported by El-Sayed $\boldsymbol{e t}$ al (2016), Emara et al (2016), Elhamamsey et al (2016), Abd El-Moneim et al (2017), Khan et al (2017) and Emara et al (2018b).
Effect of planting pattern (row spacing) on cotton yield and yield components were studied by several researches Başal et al (2014), Hamoda and Emara (2014), Wang et al (2016), Ghoprial et al (2018), Emara et al (2020) and Deshish (2021a).

Consequently, many workers came to reported that NK fertilizer levels is the most limiting factor to increase cotton yield and yield attributes by Emara $\boldsymbol{e t}$ al (2016), Elhamamsey et al (2016), Kappes et al (2016), Emara and Abd el-Aal (2017), Emara et al (2018a) and Zakzok et al (2018) and Deshish (2021b).

This study aimed to investigate the suitable agricultural management practices such as planting dates, planting patterns and $\mathrm{NK}$ fertilizer levels of new promising hybrid cotton (CB58X Giza 90).

\section{Materials and Methods}

Two field experiments were carried out in Mallawi region, Minia Governorate, during 2019 and 2020 seasons to study the effect of two planting date $\left(20^{\text {th }}\right.$ March and 20 $0^{\text {th }}$ April), four planting patterns $\{65 \mathrm{~cm}$ ridges width $+25 \mathrm{~cm}$ hills space $\left(\mathbf{P}_{1}\right), 65 \mathrm{~cm}$ ridges width $+30 \mathrm{~cm}$ hill space $\left(\mathbf{P}_{2}\right), 130 \mathrm{~cm}$ bed width +25 $\mathrm{cm}$ hill space planted in two sides $\left(\mathbf{P}_{3}\right), 130 \mathrm{~cm}$ bed width $+30 \mathrm{~cm}$ hill space planted in two sides $\left.\left(\mathbf{P}_{4}\right)\right\}$ and 
three NK fertilizer levels $\left\{45 \mathrm{~N}+24 \mathrm{~K} 2 \mathrm{O} \mathrm{kg} \mathrm{fed}^{-1}\right.$ $\left(\mathbf{N K}_{1}\right), 60 \mathrm{~N}+36 \mathrm{~K}_{2} \mathrm{O} \mathrm{kg} \mathrm{fed}{ }^{-1}\left(\mathbf{N K}_{2}\right), 75 \mathrm{~N}+48 \mathrm{~K}_{2} \mathrm{O}$ $\left.\mathrm{kg} \mathrm{fed}^{-1}\left(\mathbf{N K}_{3}\right)\right\}$ on yield and yield components of Egyptian cotton (Gossypium barbadense, L.) promising hybrid cotton (CB58 X Giza 90). The soil was clay in texture with a $\mathrm{pH}$ value of 8.14 and 8.23 , an organic matter content of 1.99 and $2.05 \%$, available $\mathrm{N}$ of 0.112 and $0.125 \%$ and available $\mathrm{K}$ of 0.330 and $0.335 \%$ during the first and second seasons, respectively. Here, it should be noted that the preceding crop was corn in the two seasons. Data in Table 1 showed the measured climatic factors (maximum air temperature ${ }^{\circ} \mathrm{C}$ (Max. Temp.), minimum air temperature ${ }^{\circ} \mathbf{C}$ (Min. Temp.), Average relative humidity \% (Avg. $\mathrm{RH})$ and Soil Temp. ${ }^{\circ} \mathrm{C}$ (Min.Temp.) during the experimental period (March till October during 2019 and 2020 seasons). These data collected from automated weather station of Mallawi location.

Table 1. Average monthly climatic data of Mallawi location during the two studied seasons of $2019 \& 2020$.

\begin{tabular}{|c|c|c|c|c|}
\hline Month & Max. Temp. ${ }^{\circ} \mathrm{C}$ & Min. Temp. ${ }^{\circ} \mathrm{C}$ & Ave. RH\% & Soil Temp. ${ }^{\circ} \mathrm{C}$ \\
\hline & \multicolumn{4}{|c|}{$\begin{array}{l}\text { First season (2019) } \\
\end{array}$} \\
\hline March & 46.2 & 19.0 & 50.8 & 37.9 \\
\hline April & 36.7 & 18.8 & 57.4 & 29.6 \\
\hline May & 46.1 & 15.9 & 38.5 & 31.6 \\
\hline June & 46.3 & 19.1 & 39.2 & 35.9 \\
\hline July & 45.9 & 19.5 & 46.2 & 37.2 \\
\hline August & 46.2 & 19.0 & 50.0 & 38.3 \\
\hline September & 42.6 & 17.1 & 47.9 & 39.2 \\
\hline \multirow[t]{2}{*}{ October } & 33.7 & 18.2 & 47.4 & 38.5 \\
\hline & \multicolumn{4}{|c|}{ Second season (2020) } \\
\hline March & 47.1 & 20.1 & 49.2 & 38.7 \\
\hline April & 36.9 & 19.4 & 58.2 & 28.8 \\
\hline May & 44.9 & $16 . .9$ & 39.3 & 32.0 \\
\hline June & 46.8 & 19.5 & 37.9 & 36.4 \\
\hline July & 44.7 & 20.0 & 45.6 & 38.3 \\
\hline August & 47.7 & 19.7 & 49.1 & 38.3 \\
\hline September & 44 & 18.1 & 48.7 & 39.4 \\
\hline October & 35.9 & 18.0 & 48.0 & 39.1 \\
\hline
\end{tabular}

The variables in each experiment were distributed as randomized complete block design (RCBD) using split split-plot arrangement with 4 replicates. Two sowing dates were arranged in the main plots, the sub plots were assigned random by to the four planting patterns randomly and the three NK fertilizers levels were arranged random in the sub sub-plots. The area of each sub sub-plot was $23.4 \mathrm{~m}^{2}$ (including 6 ridges width of ridge was $65 \mathrm{~cm}$ and the length of ridge was $6 \mathrm{~m})$. Phosphorus fertilizer was added at a levels of $22.5 \mathrm{~kg}$ after ridging and before planting in both seasons. Each season contained two separate experiments represented the two planting dates, i.e. $20^{\text {th }}$ March and $20^{\text {th }}$ April. Nitrogen fertilizer in the form of ammonium nitrate $(33.5 \% \mathrm{~N})$ at the tested levels was applied before the second and third irrigations in two equal doses, Potassium fertilizers in the form of potassium sulfate $\left(48 \% \mathrm{~K}_{2} \mathrm{O}\right)$ at the tested levels was applied before third irrigation in one doses. Other agricultural practices were done as recommended in region.

At harvest, random samples of ten plants were chosen from the inner rows of every sub sub-plot. The characters estimated on all the sampled plants at each sampling date were as follows: plant height $(\mathrm{cm})$, No. sympodial plant ${ }^{-1}$, No. open bolls plant ${ }^{-1}$, boll weight $(\mathrm{g})$, seed index $(\mathrm{g})$, lint percentage and seed cotton yield plant ${ }^{-1}$. Seed cotton yield $\left(\right.$ kentar fed $\left.^{-1}\right)$ was estimated as the weight of seed cotton yield $(\mathrm{kg})$ picked from the whole plot in each experimental unit collected from two picks, then converted to yield fed ${ }^{1}$ in kentar $(1 \mathrm{Kentar}=157.5 \mathrm{~kg}$ seed cotton $)$.

Data collected on different parameters were analyzed statistically by using MSTAT-C programme (Anonymous, 1986) for analysis of variance. Whenever, the results were found to be significant, the treatments means were compared using LSD at 0.05 level of probability (Steel et al., 1997).

\section{Results and Discussion}

\section{-Effect of planting dates:}

Data in Table 2 showed that plant height, No. sympodial plant ${ }^{-1}$, No. open bolls plant ${ }^{-1}$, boll weight, seed index, lint percentage, seed cotton yield plant ${ }^{-1}$ and seed cotton yield fed ${ }^{-1}$ were significantly affected by planting dates in two seasons. The shortest plants were produced by early planting, whereas the tallest plants were recorded by late planting date. The highest values of No. sympodial plant ${ }^{-1}$ (20.36 and 20.45), No. open bolls plant ${ }^{-1}(15.12$ and 15.62 boll), boll weight (3.01 and $2.68 \mathrm{~g}$ ), seed index (10.72 and $9.83 \mathrm{~g}$ ), lint percentage (6.67 and 7.24\%), seed cotton yield plant ${ }^{-1}$ (41.09 and $34.79 \mathrm{~g})$ and seed cotton yield fed ${ }^{-1}$ (11.74 and 8.12 kentar) in The first and second seasons, respectively. 
Table 2. Yield and its components of Egyptian cotton as affected by planting date in 2019 and 2020 seasons.

\begin{tabular}{|c|c|c|c|c|c|c|}
\hline \multirow[b]{2}{*}{ Characters } & \multicolumn{2}{|c|}{2019 season } & \multirow{2}{*}{$\begin{array}{c}\text { LSD } \\
\text { at } \\
\mathbf{5 \%}\end{array}$} & \multicolumn{2}{|c|}{2020 season } & \multirow{2}{*}{$\begin{array}{c}\text { LSD at } \\
5 \%\end{array}$} \\
\hline & $\begin{array}{c}20^{\text {th }} \\
\text { March }\end{array}$ & $\begin{array}{c}\mathbf{2 0}^{\text {th }} \\
\text { April }\end{array}$ & & $\begin{array}{c}20^{\text {th }} \\
\text { March }\end{array}$ & $\begin{array}{c}\mathbf{2 0}^{\text {th }} \\
\text { April }\end{array}$ & \\
\hline Plant height $(\mathrm{cm})$ & 138.7 & 148.9 & 0.82 & 137.1 & 140.4 & 1.43 \\
\hline No. sympodial plant ${ }^{-1}$ & 20.36 & 19.64 & 0.22 & 20.45 & 18.34 & 0.06 \\
\hline No. open bolls plant ${ }^{-1}$ & 15.12 & 12.66 & 0.85 & 15.62 & 13.81 & 0.64 \\
\hline Boll weight $(\mathrm{g})$ & 3.01 & 2.87 & 0.08 & 2.68 & 2.29 & 0.10 \\
\hline Seed index (g) & 10.72 & 10.22 & 0.07 & 9.83 & 9.41 & 0.23 \\
\hline Lint $(\%)$ & 6.67 & 6.08 & 0.12 & 7.24 & 6.63 & 0.29 \\
\hline Seed cotton yield plant ${ }^{-1}(\mathrm{~g})$ & 41.09 & 39.12 & 1.29 & 34.79 & 23.51 & 2.11 \\
\hline Seed cotton yield fed ${ }^{-1}$ (kentar) & 11.74 & 8.10 & 0.23 & 8.12 & 6.76 & 0.73 \\
\hline
\end{tabular}

The results indicated that late sown plants grew faster than early sown ones, which is evident that higher temperature provided by late sowing enhanced stem elongation of cotton plants. Cotton growth increases linearly as temperature increases. Similar results were obtained by Hamed (2012), Deshish (2013), Deho et al (2014), Mohamed et al (2016), Abd El-Moneim et al (2017), Emara et al (2018b) and Anwar et al (2020).

\section{-Effect of planting patterns:}

Data in Table 3 showed that plant height, No. sympodial plant $^{-1}$, No. open bolls plant ${ }^{-1}$, boll weight, seed index, lint percentage, seed cotton yield plant ${ }^{-1}$ and seed cotton yield fed ${ }^{-1}$ were significantly affected by planting patterns in two seasons. The highest values of plant height (146.49 and 141.0) in the first and second seasons, respectively obtained by $\mathrm{P} 1$ treatment. While, the highest values of No. sympodial plant $^{-1}$ (20.33 and 20.03) in the first and second seasons, respectively by $\mathrm{P} 2$ treatment. Moreover, the highest values of No. open bolls plant ${ }^{-1}$ (14.20 and15.16 boll), boll weight (3.12 and $2.77 \mathrm{~g}$ ), seed index (10.73 and $9.95 \mathrm{~g})$, lint percentage $(6.71$ and $7.29 \%$ ), seed cotton yield plant ${ }^{-1}$ (40.11 and $32.48 \mathrm{~g}$ ) and seed cotton yield fed ${ }^{-1}$ (10.16 and 7.94 kentar) in the first and second seasons, respectively obtained by P4 treatment. The narrow ridges decrease the yield contributing traits and ultimately the seed cotton yield and when the cotton was sown in wider ridges, plants were able to receive an additional benefit of soil moisture and nutrients during the growing season,

Table 3. Yield and its components of Egyptian cotton as affected by planting patterns in 2019 and 2020 seasons

\begin{tabular}{|c|c|c|c|c|c|}
\hline \multirow[b]{2}{*}{ Characters } & \multicolumn{4}{|c|}{2019 season } & \multirow{2}{*}{$\begin{array}{c}\text { LSD at } \\
5 \%\end{array}$} \\
\hline & P1 & P2 & P3 & P4 & \\
\hline Plant height $(\mathrm{cm})$ & 146.49 & 142.70 & 144.52 & 141.58 & 1.08 \\
\hline No. sympodial plant ${ }^{-1}$ & 19.53 & 20.33 & 19.89 & 20.24 & 0.25 \\
\hline No. open bolls plant ${ }^{-1}$ & 13.43 & 13.80 & 14.14 & 14.20 & 0.35 \\
\hline Boll weight $(\mathrm{g})$ & 2.77 & 2.99 & 2.87 & 3.12 & 0.09 \\
\hline Seed index (g) & 10.23 & 10.50 & 10.43 & 10.73 & 0.14 \\
\hline Lint $(\%)$ & 6.08 & 6.42 & 6.27 & 6.71 & 0.16 \\
\hline Seed cotton yield plant ${ }^{-1}(\mathrm{~g})$ & 30.45 & 36.44 & 33.37 & 40.11 & 0.99 \\
\hline \multirow[t]{2}{*}{ Seed cotton yield fed ${ }^{-1}$ (kentar) } & 9.70 & 10.15 & 9.68 & 10.16 & 0.22 \\
\hline & \multicolumn{4}{|c|}{2020 season } & \\
\hline Plant height $(\mathrm{cm})$ & 141.0 & 138.4 & 140.0 & 135.5 & 0.90 \\
\hline No. sympodial plant ${ }^{-1}$ & 18.71 & 20.03 & 19.28 & 19.55 & 0.40 \\
\hline No. open bolls plant ${ }^{-1}$ & 14.19 & 14.85 & 14.66 & 15.16 & 0.31 \\
\hline Boll weight (g) & 2.26 & 2.53 & 2.39 & 2.77 & 0.12 \\
\hline Seed index $(g)$ & 9.36 & 9.72 & 9.46 & 9.95 & 0.15 \\
\hline Lint $(\%)$ & 6.65 & 7.03 & 6.78 & 7.29 & 0.09 \\
\hline Seed cotton yield plant ${ }^{-1}(\mathrm{~g})$ & 25.75 & 30.68 & 27.68 & 32.48 & 1.10 \\
\hline Seed cotton yield fed ${ }^{-1}$ (kentar) & 7.10 & 7.56 & 7.18 & 7.94 & 0.28 \\
\hline
\end{tabular}

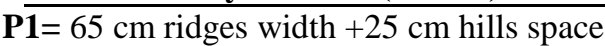

$\mathbf{P 3}=130 \mathrm{~cm}$ bed width $+25 \mathrm{~cm}$ hill space planted in two sides

$\mathbf{P 4}=130 \mathrm{~cm}$ bed width $+30 \mathrm{~cm}$ hill space planted in two sides

which allowed more flower buds to form, which resulted in additional bolls reaching maturation and an increase in the distance between plants within the row will minimize the effect of plant shading and as aftermath, crop yield will improve. This results are in harmony with those obtained by Baumhardt et al
(2018), Ghoprial et al (2018), Emara et al (2020), Deshish (2021a) and Deshish (2021b).

\section{-Effect of NK fertilizer levels:}

Data in Table 4 showed that NK fertilizer levels had a significant effect on all studied characters 
in both seasons. NK3 treatment gave the highest values of plant height, No. sympodial plant ${ }^{-1}$, No. open bolls plant ${ }^{-1}$, boll weight, seed index, lint percentage, seed cotton yield plant ${ }^{-1}$ and seed cotton yield $\mathrm{fed}^{-1}$ in the first and second seasons. These results could be attributed to the stimulation effect of
NK on the formation of more sound seeds with a heavyweight. Similar results were obtained by Emara and Abd el-Aal (2017), MANJUNATHA et al (2017), Zakzok et al (2018), Emara et al (2018a), Emara et al (2020), Deshish (2021a), and Deshish (2021b).

Table 4. Yield and its components of Egyptian cotton as affected by NK fertilizer levels in 2019 and 2020 seasons

\begin{tabular}{|c|c|c|c|c|c|c|c|c|}
\hline \multirow[b]{2}{*}{ Characters } & \multicolumn{3}{|c|}{2019 season } & \multirow{2}{*}{$\begin{array}{c}\text { LSD } \\
\text { at 5\% }\end{array}$} & \multicolumn{3}{|c|}{2020 season } & \multirow{2}{*}{$\begin{array}{c}\text { LSD } \\
\text { at } 5 \%\end{array}$} \\
\hline & NK1 & NK2 & NK3 & & NK1 & NK2 & NK3 & \\
\hline Plant height $(\mathrm{cm})$ & 140.4 & 143.9 & 147.1 & 1.15 & 136.5 & 139.2 & 140.5 & 1.36 \\
\hline No. fruiting branches plant ${ }^{-1}$ & 19.38 & 20.08 & 20.53 & 0.34 & 18.60 & 19.49 & 20.08 & 0.30 \\
\hline No. open bolls plant ${ }^{-1}$ & 12.33 & 14.34 & 15.01 & 0.75 & 13.47 & 14.85 & 15.82 & 0.28 \\
\hline Boll weight (g) & 2.81 & 2.96 & 3.04 & 0.07 & 2.33 & 2.53 & 2.61 & 0.09 \\
\hline Seed index $(g)$ & 10.29 & 10.48 & 10.64 & 0.14 & 9.38 & 9.67 & 9.81 & 0.15 \\
\hline Lint $(\%)$ & 37.47 & 37.82 & 38.09 & NS & 41.37 & 41.98 & 42.21 & 0.37 \\
\hline Seed cotton yield plant ${ }^{-1}(\mathrm{~g})$ & 30.76 & 36.04 & 38.47 & 1.72 & 25.23 & 29.97 & 32.24 & 2.12 \\
\hline Seed cotton yield fed ${ }^{-1}$ (kentar) & 9.58 & 9.97 & 10.21 & 0.30 & 7.12 & 7.49 & 7.71 & 0.30 \\
\hline
\end{tabular}

NK1=45 kg N + $24 \mathrm{~kg} \mathrm{~K}_{2} \mathrm{O} \mathrm{fed}^{-1} \quad$ NK2=60 kg N + 36 kg K $2 \mathrm{O}_{\text {fed }}{ }^{-1} \quad \mathbf{N K 3}=75 \mathrm{~kg} \mathrm{~N}+48 \mathrm{~kg} \mathrm{~K}_{2} \mathrm{O} \mathrm{fed}^{-1} \quad$ NS=No significance

\section{-Effect of the interactions:}

Boll weight, seed index, seed cotton yield plant $^{-1}$ and seed cotton yield fed ${ }^{-1}$ were affected by the interaction between planting date and planting patterns in the first season (Table 5A). Sown in $20^{\text {th }}$ March under P4 treatment gave the highest values of boll weight $(3.27 \mathrm{~g})$, seed index $(10.89 \mathrm{~g})$, seed cotton yield plant ${ }^{-1}(39.64 \mathrm{~g})$ and seed cotton yield fed ${ }^{-1}$ (12.19 kentar) in the first season. On the other hand, the lowest values of boll weight, seed index, seed cotton yield plant ${ }^{-1}$ and seed cotton yield fed ${ }^{-1}$ were obtained by sown in $20^{\text {th }}$ April under P1 treatment in both seasons. Concerning, plant height and No. open bolls plant ${ }^{-1}$ were affected by the interaction between planting date and NK fertilizer levels in the first and second seasons, respectively (Table 5B). The tallest plants $(153.94 \mathrm{~cm})$ of cotton produced by sown in $20^{\text {th }}$ April under NK3 treatment, while sown in $20^{\text {th }}$ March under NK3 treatment gave the maximum value of No. open bolls plant ${ }^{-1}$. On the other hand, the lowest value of plant height gave by sown in $20^{\text {th }}$ March under NK1 treatment and the minimum value of No. open bolls plant $^{-1}$ obtained by sown in $20^{\text {th }}$ April under NK1 treatment.

Table 5A. Effect of the interaction between of planting date and patterns on some yield and its components in 2019 and 2020 seasons

\begin{tabular}{|c|c|c|c|c|c|c|c|c|}
\hline \multirow{3}{*}{ Planting date } & \multicolumn{4}{|c|}{2019 season } & \multicolumn{4}{|c|}{2020 season } \\
\hline & \multicolumn{8}{|c|}{ Planting patterns } \\
\hline & P1 & $\mathbf{P 2}$ & P3 & P4 & P1 & $\mathbf{P 2}$ & P3 & $\mathbf{P 4}$ \\
\hline & \multicolumn{8}{|c|}{ Boll weight (g) } \\
\hline 20 ${ }^{\text {th }}$ March & 2.81 & 3.09 & 2.87 & 3.27 & 2.46 & 2.72 & 2.59 & 2.96 \\
\hline 20 ${ }^{\text {th }}$ April & 2.73 & 2.90 & 2.87 & 2.97 & 2.06 & 2.35 & 2.19 & 2.58 \\
\hline L.S.D at $5 \%$ & \multicolumn{4}{|c|}{0.12} & \multicolumn{4}{|c|}{ NS } \\
\hline & \multicolumn{8}{|c|}{ Seed index (g) } \\
\hline $20^{\text {th }}$ March & 10.63 & 10.65 & 10.72 & 10.89 & 9.51 & 10.06 & 9.61 & 10.16 \\
\hline 20 ${ }^{\text {th }}$ April & 9.82 & 10.34 & 10.14 & 10.56 & 9.22 & 9.37 & 9.31 & 9.74 \\
\hline L.S.D at $5 \%$ & \multicolumn{4}{|c|}{0.20} & \multicolumn{4}{|c|}{ NS } \\
\hline & \multicolumn{8}{|c|}{ Seed cotton yield plant $\mathbf{t}^{-1}(\mathrm{~g})$} \\
\hline $20^{\text {th }}$ March & 31.88 & 38.05 & 34.77 & 41.09 & 30.00 & 36.40 & 33.11 & 39.64 \\
\hline 20 ${ }^{\text {th }}$ April & 36.45 & 29.02 & 34.84 & 31.97 & 21.49 & 24.97 & 22.25 & 25.32 \\
\hline L.S.D at $5 \%$ & \multicolumn{4}{|c|}{ NS } & \multicolumn{4}{|c|}{1.56} \\
\hline & \multicolumn{8}{|c|}{ Seed cotton yield fed ${ }^{-1}$ (kentar) } \\
\hline 20 ${ }^{\text {th }}$ March & 11.60 & 12.19 & 11.48 & 11.69 & 7.84 & 8.22 & 7.83 & 8.60 \\
\hline 20 ${ }^{\text {th }}$ April & 7.80 & 8.10 & 7.88 & 8.63 & 6.35 & 6.89 & 6.52 & 7.27 \\
\hline L.S.D at $5 \%$ & \multicolumn{4}{|c|}{0.31} & \multicolumn{4}{|c|}{ NS } \\
\hline
\end{tabular}


Table 5B. Effect of the interaction between planting date and NK fertilizer levels on plant height and No. open bolls plant ${ }^{-1}$ in 2019 and 2020 seasons

\begin{tabular}{|c|c|c|c|c|c|c|}
\hline \multirow[t]{2}{*}{ Planting date } & \multicolumn{2}{|c|}{2019 season } & \multicolumn{3}{|c|}{$\begin{array}{c}2020 \text { season } \\
\text { NK fertilizer levels kg } \text { fed }^{-1}\end{array}$} & \multirow[b]{2}{*}{ NK3 } \\
\hline & NK1 & NK2 & NK3 & NK1 & NK2 & \\
\hline & \multicolumn{5}{|c|}{ Plant height $(\mathrm{cm})$} & \\
\hline $20^{\text {th }}$ March & 136.97 & 138.85 & 140.39 & 135.15 & 137.31 & 138.84 \\
\hline 20 ${ }^{\text {th }}$ April & 143.84 & 148.95 & 153.94 & 137.89 & 141.13 & 142.29 \\
\hline \multirow[t]{2}{*}{ L.S.D at $5 \%$} & 1.63 & & & NS & & \\
\hline & \multicolumn{5}{|c|}{ No. open bolls plant ${ }^{-1}$} & \\
\hline $20^{\text {th }}$ March & 13.41 & 15.45 & 16.51 & 14.38 & 15.73 & 16.74 \\
\hline 20 ${ }^{\text {th }}$ April & 11.24 & 13.23 & 13.51 & 12.56 & 13.98 & 14.91 \\
\hline L.S.D at $5 \%$ & \multicolumn{3}{|c|}{ NS } & \multicolumn{3}{|c|}{0.40} \\
\hline
\end{tabular}

NK1=45 kg N + 24 kg K $\mathrm{O} \mathrm{fed}^{-1} \quad$ NK2=60 kg N + $36 \mathrm{~kg} \mathrm{~K}_{2} \mathrm{O} \mathrm{fed}^{-1} \quad \mathbf{N K 3 = 7 5 ~ k g ~ N ~ + ~} 48 \mathrm{~kg} \mathrm{~K}_{2} \mathrm{O}$ fed ${ }^{-1} \quad$ NS=No significance

Regarding, plant height and No. open bolls plant ${ }^{-1}$ were affected by the interaction between planting patterns and $\mathrm{NK}$ fertilizer levels in the first and second seasons, respectively (Table 5C). The highest value of plant height gave by $\mathrm{P} 1$ treatment under NK3 treatment, whereas, the highest No. open bolls plant ${ }^{-1}$ produced by $\mathrm{P} 2$ treatment under NK3 treatment. On the contrary, the lowest values of plant height and No. open bolls plant ${ }^{-1}$ were obtained by NK1 treatment under $\mathrm{P} 2$ and $\mathrm{P} 1$ treatments, respectively.

Table 5C. Effect of the interaction between planting patterns and NK fertilizer levels on plant height and No. open bolls plant ${ }^{-1}$ in 2019 and 2020 seasons

\begin{tabular}{|c|c|c|c|c|c|c|}
\hline \multirow{4}{*}{$\begin{array}{l}\text { Planting } \\
\text { patterns }\end{array}$} & \multicolumn{3}{|c|}{2019 season } & \multicolumn{3}{|c|}{2020 season } \\
\hline & \multicolumn{6}{|c|}{$\begin{array}{l}\text { NK fertilizer levels kg fed } \\
\text {-1 }^{-1}\end{array}$} \\
\hline & NK1 & NK2 & NK3 & NK1 & NK2 & NK3 \\
\hline & \multicolumn{6}{|c|}{ Plant height $(\mathrm{cm})$} \\
\hline P1 & 143.13 & 146.85 & 149.50 & 138.24 & 141.75 & 143.04 \\
\hline $\mathbf{P 2}$ & 137.00 & 144.31 & 146.78 & 135.45 & 139.66 & 140.25 \\
\hline P3 & 141.69 & 143.88 & 148.00 & 137.28 & 139.86 & 142.96 \\
\hline P4 & 139.81 & 140.56 & 144.38 & 135.13 & 135.60 & 136.01 \\
\hline \multirow[t]{2}{*}{ L.S.D at $5 \%$} & & 2.30 & & & NS & \\
\hline & \multicolumn{6}{|c|}{ No. open bolls plant ${ }^{-1}$} \\
\hline P1 & 11.88 & 14.06 & 14.36 & 12.71 & 14.36 & 15.49 \\
\hline $\mathbf{P 2}$ & 12.69 & 13.98 & 14.74 & 13.24 & 14.59 & 16.74 \\
\hline P3 & 12.30 & 14.86 & 15.24 & 13.54 & 15.04 & 15.41 \\
\hline P4 & 12.44 & 14.46 & 15.69 & 14.39 & 15.43 & 15.66 \\
\hline L.S.D at $5 \%$ & \multicolumn{3}{|c|}{ NS } & \multicolumn{3}{|c|}{0.56} \\
\hline
\end{tabular}

$\mathbf{P 1}=65 \mathrm{~cm}$ ridges width $+25 \mathrm{~cm}$ hills space $\quad$ P2 $=65 \mathrm{~cm}$ ridges width $+30 \mathrm{~cm}$ hill space $\quad$ NS=No significance $\mathbf{P 3}=130 \mathrm{~cm}$ bed width $+25 \mathrm{~cm}$ hill space planted in two sides $\mathbf{P 4}=130 \mathrm{~cm}$ bed width $+30 \mathrm{~cm}$ hill space planted in two sides

NK1=45 kg N + $24 \mathrm{~kg} \mathrm{~K}_{2} \mathrm{O} \mathrm{fed}^{-1} \quad$ NK2=60 kg N + $36 \mathrm{~kg} \mathrm{~K}_{2} \mathrm{O} \mathrm{fed}^{-1} \quad \mathbf{N K 3 = 7 5 ~ k g ~ N ~}+48 \mathrm{~kg} \mathrm{~K}_{2} \mathrm{O}$ fed $^{-1}$

Regarding the interaction effect, among planting date, planting patterns and $\mathrm{NK}$ fertilizer levels were significant differences of No. sympodial plant ${ }^{-1}$ in the first season only (Table 6). The highest value of No. sympodial plant ${ }^{-1}$ produced by sown in $20^{\text {th }}$ March with $\mathrm{P} 2$ treatment under NK3 treatment. While, sown in $20^{\text {th }}$ April with P3 treatment under NK1 treatment gave the lowest value of No. sympodial plant ${ }^{-1}$. 
Table 6. Effect of the interaction between planting date, planting patterns and NK fertilizer levels on No. sympodial plant ${ }^{-1}$ in 2019 and 2020 seasons

\begin{tabular}{|c|c|c|c|c|c|c|c|}
\hline \multirow{3}{*}{$\begin{array}{c}\text { Planting } \\
\text { date }\end{array}$} & \multirow{3}{*}{$\begin{array}{l}\text { Planting } \\
\text { patterns }\end{array}$} & \multicolumn{3}{|c|}{2019 season } & \multicolumn{3}{|c|}{2020 season } \\
\hline & & \multicolumn{6}{|c|}{ NK fertilizer levels kg fed ${ }^{-1}$} \\
\hline & & NK1 & NK2 & NK3 & NK1 & NK2 & NK3 \\
\hline \multirow{4}{*}{$20^{\text {th }}$ March } & P1 & 19.48 & 19.70 & 20.03 & 19.15 & 19.96 & 20.40 \\
\hline & $\mathbf{P 2}$ & 19.90 & 20.68 & 21.88 & 20.08 & 20.90 & 22.03 \\
\hline & P3 & 19.70 & 20.38 & 20.88 & 19.75 & 20.40 & 20.88 \\
\hline & P4 & 19.78 & 20.58 & 21.33 & 19.63 & 20.68 & 21.50 \\
\hline \multirow{4}{*}{$2^{\text {th }}$ April } & P1 & 18.15 & 20.63 & 19.23 & 16.93 & 17.73 & 18.08 \\
\hline & $\mathbf{P 2}$ & 19.70 & 19.68 & 20.13 & 18.15 & 19.08 & 19.95 \\
\hline & P3 & 19.03 & 19.23 & 20.13 & 17.65 & 18.38 & 18.63 \\
\hline & P4 & 19.30 & 19.83 & 20.65 & 17.50 & 18.78 & 19.21 \\
\hline \multicolumn{2}{|c|}{$\begin{array}{l}\text { L.S.D at 5\% } \\
\mathbf{P 1}=65 \mathrm{~cm} \text { ridges width }+25 \mathrm{~cm} \text { hills space }\end{array}$} & \multicolumn{3}{|c|}{0.97} & \multicolumn{3}{|c|}{ NS } \\
\hline \multicolumn{8}{|c|}{$\begin{array}{l}\mathbf{P 3}=130 \mathrm{~cm} \text { bed width }+25 \mathrm{~cm} \text { hill space planted in two sides } \mathbf{P 4}=130 \mathrm{~cm} \text { bed width }+30 \mathrm{~cm} \text { hill space planted in two sides } \\
\text { NK1 }=45 \mathrm{~kg} \mathrm{~N}+24 \mathrm{~kg} \mathrm{~K}_{2} \mathrm{O} \mathrm{fed}^{-1} \quad \mathbf{N K 2}=60 \mathrm{~kg} \mathrm{~N}+36 \mathrm{~kg} \mathrm{~K}_{2} \mathrm{O}^{-1} \quad \mathbf{N K 3}=75 \mathrm{~kg} \mathrm{~N}+48 \mathrm{~kg} \mathrm{~K}_{2} \mathrm{O} \mathrm{fed}^{-1} \\
\text { It can be concluded that early planting, } 130 \mathrm{~cm} \text { bed width }+30 \mathrm{~cm}^{-1} \text { hill space planted in two sides and fertilized by } 75 \mathrm{~kg} \mathrm{~N}+48 \mathrm{~kg} \mathrm{~K} 2 \mathrm{O} \text { fe } \\
1 \text { under the conditions of the experiment. }\end{array}$} \\
\hline
\end{tabular}

\section{References}

Abd El-Moneim M. H., M. A. Omar and S. S. ELTabbakh, A. I. Nawar 2017. The effect of date and pattern of sowing on growth, productivity and technological characters of cotton (Gossypium barbadense 1.) Variety Giza 86. Alex. Sci. exchange J., 38(3): 389-396.

Anonymous (1986). MSTATC, Microcomputer Statistical Programme. Michigan State University, Michigan, Lansing, USA.

Anwar M. R., B. Wang, D. L. Liu and C. Waters, 2020. Late planting has great potential to mitigate the effects of future climate change on Australian rain-fed cotton. Sci.of the Total Environment 714 (136806):1-13.

Başal, H., V. Sezener, O. Canavar, K. Kızılkaya, and N. Dağdelen. 2014. Effects of water stress and plant density on cotton (Gossypium hirsutum, L.) cultivars differing in maturity and seed size: I. yield components and fiber quality parameters. Inter. J. Agric. Innov. Res., 3 (3): 755-760.

Baumhardt R. L., R. C. Schwartz, G. W. Marek and J. M. Bell, 2018. Planting geometry effects on the growth and yield of dryland cotton. Agric. Sci., (9): 99-116.

Deho, Z. A.; S. Tunio; Q.Chachar and F. C. Oad 2014. Impact of sowing dates and picking stages on yield and seed maturity of cotton (Gossypium hirsutum L.) varieties. Sarhad Journal of Agriculture, 30 (4): 404: 410.

Deshish El-D. El-D., 2013. Suitable agricultural managment practices for the new promising hybrid cotton [giza 84 (giaz 70 x 51b)] pima62. J. Plant Production, Mansoura Univ., 4 (12):1885 - 1896.

Deshish El-D. El-D., 2021a. Effect of plants distribution systems on growth, yield and quality of cotton variety Giza 96 under different levels of NPK fertilization. J. of Plant Production, Mansoura Univ. 12 (3):243-248.
Deshish El-D. El-D., 2021b. Effect of row width and spacing between hills on growth, yield and quality of cotton variety Giza 96 under different levels of NPK fertilization. J. of Plant Production, Mansoura Univ. 12 (3):225-230.

Elhamamsey, M. H., E. M. Shalaby, E. A. Ali and M. A. Emara, 2016. Effect of some cultural practices on shedding and yield of Egyptian cotton. Assiut J. Agric. Sci., 47 (4):41-51.

El-Sayed, A. E. M., E. M. M. Shalaby, W. M. ElShazly and M. T. Said, 2016. Study of Egyptian cotton to alternative irrigation systems with NPK rates and frequencies under two planting dates. Assiut J. Agric. Sci., 47 (4):52-71.

Emara M. A. A. and A. S. Abdel-Aal, 2017. Effect of nano-fertilizer on productivity of cotton under nutrient stress conditions. Egy. J. of Appl. Sci., 32 (12 B): 445-458.

Emara M. A. A., A. S. Abdel-Aal, R. M. Hassan and A. E. El-Gabiery, 2016. Effect of sowing dates and bio-fertilizer under different NPK fertile levels on growth, yield and fiber of promising hybrid cotton Giza 86 X 10229. Egypt. J. of Appl. Sci., 31(12): $357-376$.

Emara M. A. A., S. A. F. Hamoda and Maha M.A. Hamada, 2018a. Effect of potassium silicate and NPK fertilization levels on cotton growth and productivity under different sowing dates. Egy. $J$. Agron. The $15^{\text {th }}$ Int. Conf. Crop Sci., pp. 115-123.

Emara M. A. A., S. A. F. Hamoda and Maha M. A. Hamada, 2018b. Effect of nano-fertilizer and Nfertilization levels on productivity of Egyptian cotton under different sowing dates. Egypt. J. Agron. The 15th Int. Conf. Crop Sci., pp. 125 137.

Emara M. A., A. S. Abdel-Aal and A. A. ElHendawy, 2020. Effect of planting patterns and fertilization levels on growth, yield, and quality of cotton variety Giza 94. J. Agric. Env. Sci. Damanhour Univ. 19(2): 1-17. 
Ghoprial M. A., S. A. Sedhom, S. A. S. Mohasen, and F. S. Hamed, and El-Saeed M. M. ElGedwy, 2018. Effect of population density and nitrogen fertilizer levels on growth, yield components, yield, and fiber properties of Egyptian cotton (Giza 95). Annals of Agric. Sci., Moshtohor, 56 (1):1-13.

Hamed F. S. 2012. Response of Giza 90 cotton cultivar to sowing dates and first irrigation (almohayat) time. J. Plant Production, Mansoura Univ., 3 (12): 3027-3035.

Hamoda S. A. and M. A. Emara, 2014. Effect of planting patterns and fertilization under two planting dates on growth, yield and quality for new hybrid cotton [Giza 83 (Giza 75 x 5844)] x Giza 80. The 1st International Cotton Conference "Challenges to sustainable cotton production \& quality ". Giza, Egypt. Feb., 25 - 26th, 2014. pp: $26-27$.

Kappes K., L. Zancanaro, and E. A. B. Francisco, 2016. Nitrogen and potassium in narrow-row cotton. Rev Bras Cienc Solo v40:e0150103:1-17.

Khan A., L. Wang, S. Ali, S. A. Tung, A. Hafeez and G. Yang 2017. Optimal planting density and sowing date can improve cotton yield by maintaining reproductive organ biomass and enhancing potassium uptake. Field Crops Research (214): 164-174.
Manjunatha S. B., D. P. BIradar and Y. Aladakatti 2017. Effect of nitrogen levels and $\mathrm{K}: \mathrm{N}$ ratios on growth, yield an economics of Bt cotton. J. Farm Sci., 30 (3): (338-342).

Mohamed K. A., S. O. Yagoub, and A. K. Abd Elsalam, and A. I. Abuali, 2016. Response of sowing dates, cultivars and nitrogen application on growth, yield and oil contents of cotton crop (Gossypium hirsutum 1). Sch. J. Agric. Vet. Sci; 3(5):351-357.

Sawan, Z.M., S.A. Hafez, A.E. Basyony and A.R. Alkassas, 2006. Cottonseed, protein, oil yields and oil properties as affected by nitrogen fertilization and foliar application of potassium and a plant growth retardant. World J. Agric. Sci., 2(1):56-65.

Steel, R. G. D.; J. H. Torrie and D. A. Dickey (1997). Principles and Procedures of Statistics: A Biometrical Approach. 3rd Ed., McGraw Hill Book Co. Inc. New York. 400-428 p.

Wang, X., Y. Hou, M. Du, D. Xu, H. Lu, X. Tian, and Z. Li. 2016. Effect of planting date and plant density on cotton traits as relating to mechanical harvesting in the Yellow River valley region of China. Field Crops Res., 198: 112-121.

Zakzok A. K., R. Th. Abdrabou, A. S. Arafa and G. A. A. Abd-Elsamad, 2018. Response of cotton yield and lint properties to mineral N.P.K nanofertilization. J. Agric. Sci., 26(2): 1029 -1039. 
تأثير نظم الزراعة ومستويات التسميد النيتروجيني والبوتاسي تحت ميعادي زراعه علي المحصول ومكوناته للقطن

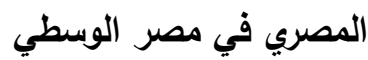

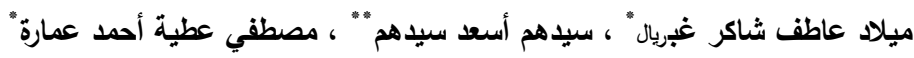

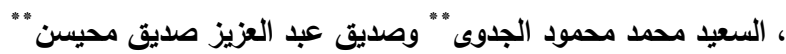

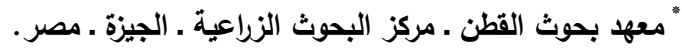

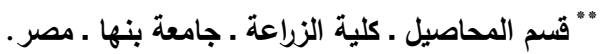

أجريت هذه الدراسة بمنطقه ملوي - محافظة المنيا خلال موسمي الزراعة 2019 و2020 لدراسة نأثير أربعة نظم الزراعة وثناث

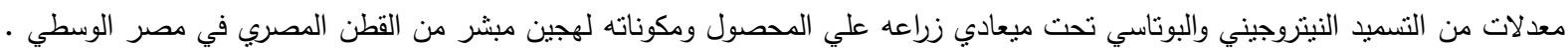

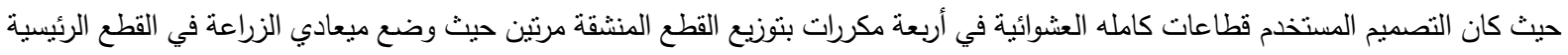

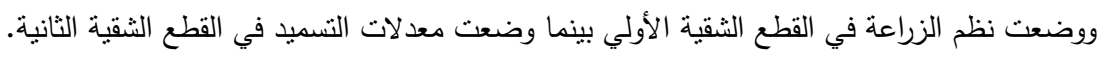

ويمكن تلخيص أهم النتائج فيما يلي:

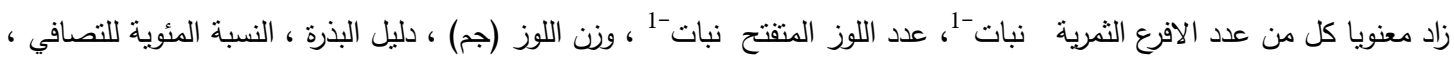

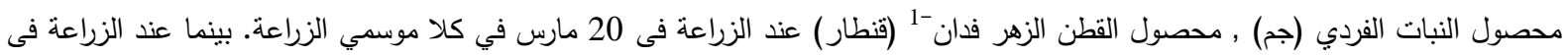
20 إبريل كانت هناك زياده معنويه في ارتفاع النبات في كلا الموسمين.

كان لنظم الزراعة تأثير معنوي لكل الصفات تحت الدراسة في كلا موسمي الزراعة. حيث كانت هناك زيادة معنوية لـعاملة الزراعة علي

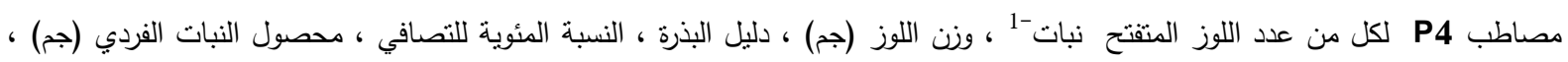
محصول القطن الزهر فدان 1-1 (قنطار) مقارنة بمعاملات نظم الزراعة الأخرى للموسمين الزراعيين.

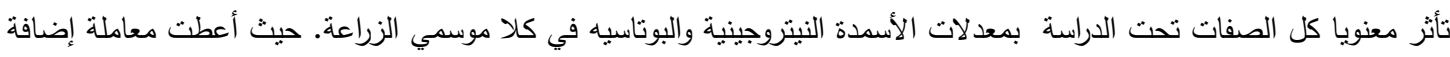

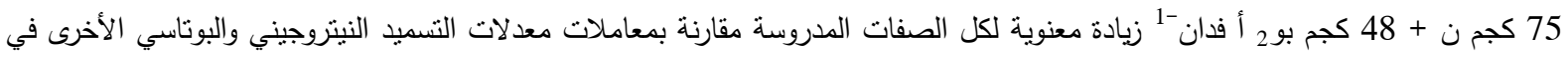
موسمي الزراعة. تأثر معنويا وزن اللوزة ، دليل البذرة ، محصول القطن الزهر فـ-1 في الموسم الأول فقط بينما محصول النبات الفردي في الموسم الثاني

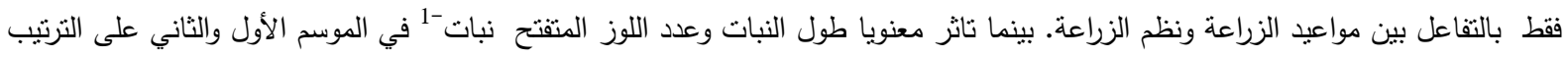

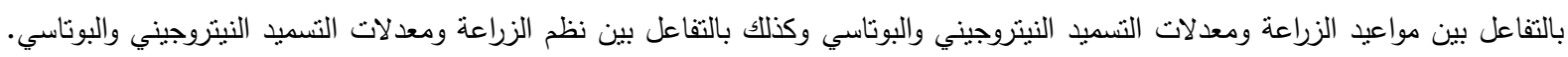

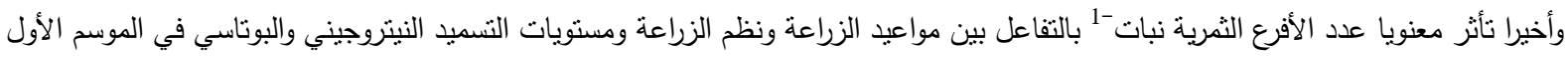

توصي هذه الدراسة بالزراعة في ميعاد 20 مارس والزراعة علي مصاطب مع التسميد بمعدل 75كجم نيتروجين+48كجم بوتاسيوم ف-1. 